\title{
Beiträge zur Kenntnis der Celluloseverdauung im Blinddarm und des Enzymgehaltes des Caecalsekretes. \\ Von \\ Arthur Scheunert.
}

(Aus der physiologisch-chemischen Abteilung der kgl. tierärztlichen Hochschule zu Dresden.) (Geh. Med.-Rat Prof. Dr. Ellenberger.)

(Der Redaktion zugegangen am 29. März 1906.)

Die Untersuchungen, welche von Ustjanzew ${ }^{1}$ ) im Zuntzschen Institut unter Zuntz' Leitung vor kurzer Zeit über die Bedeutung des Blinddarms des Kaninchens vorgenommen worden sind, haben unsere Kenntnis vom Orte der Celluloseverdauung erweitert und die Aufmerksamkeit von neuem auf die Frage der Celluloseverdauung gelenkt.

Die Tatsache, daß gewisse Tiere, und zwar speziell die Wiederkäuer, Cellulose verdauen, ist, wie bekannt, zuerst in unserem Institute 1855 durch Haubner ${ }^{8}$ ) festgestellt worden. Später haben sich verschiedene Forscher (Henneberg, Stohmann, Hoppe-Seyler, Tappeiner, Weiske u. a. m.) mit dieser Frage beschäftigt; auch in unserem Institute sind immer von neuem Untersuchungen über die Celluloseverdauung bei den verschiedenen Haustieren angestellt worden. Es wurde dabei auch versucht, die Frage zu lösen, wo, d. h. in welchem Abschnitte des Verdauungskanales die Lösung der Cellulose stattfindet. So sind Ellenberger ${ }^{3}$ ) und sein Mitarbeiter V. Hofmeister zu der Überzeugung gekommen, daß beim Pferde die Celluloseverdauung vorwiegend im Caecum, daneben aber auch im großen Colon abläuft.

1) Archiv für (Anat. u.) Physiol., 1905, S. 403.

2) Zeitschrift für deutsche Landwirte, 1855, S. 177.

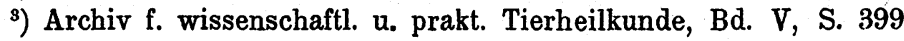
und Bd. VII-XII. 
Auch bei den anderen Säugetieren mit großem Caecum sehen sie dieses als den hauptsächlichsten Ort der Celluloseverdauung an, während bei den Wiederkäuern die Vormägen für diese Vorgänge von größerer Bedeutung sind, als das Caecum. In neuester Zeit scheinen seit einer längeren Reihe von Jahren Untersuchungen über die hier zu behandelnde Frage nicht gemacht worden $\mathrm{zu}$ sein, wenigstens fehlt es an einschlägigen Publikationen, und so ist bis vor kurzem noch keine Bestätigung der Ellenbergerschen Anschauung über den Ort der Celluloseverdauung beim Pferde erfolgt. Verschiedene, in neuerer Zeit erschienene Arbeiten über die Funktionen des Blinddarmes enthalten hierüber irgendwie entscheidende Angaben nicht.

Erst die neuesten Untersuchungen von $\mathrm{Zuntz}$ und Ustjanzew, welche eine Nachprüfung der Versuche von Bergmann und Huetgren ${ }^{1}$ ) über die Funktionen des Caecums des Kaninchens bezwecken, haben für die Ellenbergersche Ansicht eine neue Stütze geliefert.

Ustjanzew fand, daß bei Kaninchen mit ausgeschaltetem Blinddarm die Verdauung der Cellulose eine viel geringere war, als bei Tieren mit Blinddarm, an denen Kontrollversuche unter sonst gleichen Bedingungen vorgenommen wurden. Dadurch ist einwandfrei bewiesen, daß das Caecum des Kaninchens eine große Bedeutung für die Celluloseverdauung besitzt und als Hauptort derselben anzusehen ist, und es liegt die Annahme nahe, daß auch beim Pferde, bezw. allen Einhufern, die, wie das Kaninchen, ein unverhältnismäßig großes und eigenartig eingerichtetes Caecum haben, die Celluloseverdauung vorwiegend in diesem Darmabschnitte abläuft und somit die Ellenbergersche Anschauung zutreffend ist.

Über die Verdauung von Cellulose durch die Caecalflüssigkeit des'Pferdes, Schweines und Kaninchens habe ich in den letzten Jahren, sowohl bei Gelegenheit anderer Untersuchungen Beobachtungen gemacht, wie auch lediglich $\mathrm{zu}$ dem fraglichen Zwecke Versuche angestellt, deren Ergebnisse zu veröffentlichen, mir schon im Hinblick auf die neuesten Beobachtungen von Zuntz und Ustjanzew angezeigt erscheint.

1) Skand. Arch. f. Physiologie, Bd. XIV, S. 188. 
Zugleich aber möchte ich mit diesen Mitteilungen andere verbinden, die sich auf den Enzymgehalt und die verdauende Kraft der Caecalflüssigkeit (der aus dem Caecalinhalte durch Kolieren und Filtrieren zu gewinnenden Flüssigkeit) und des Caecalsekretes (bzw. der Extrakte der Caecalschleimhaut) beziehen.

I. Untersuchungen über die Celluloseverdaung im Caecum und über den Enzymgehalt und die verdauende Kraft der Caecalflüssigkeit.

Früher ist in unserem Institute festgestellt worden, daß die Caecalflüssigkeit des Pferdes recht beträchtliche Mengen von Cellulose $z u$ lösen vermag, gleichviel ob dieselbe aus Heu oder Gras hergestellt oder als Papiercellulose aus einer Fabrik bezogen worden war. Alle Versuche, die darauf hinzielten, ein celluloselösendes, cytohydrolytisches Enzym, etwa durch Fällen mit Alkohol aus der Caecalflüssigkeit $\mathrm{zu}$ isolieren, schlugen fehl, hingegen war es möglich, der Caecalflüssigkeit ihre Wirksamkeit auf Cellulose dadurch zu nehmen, daß man sie einige Minuten zum Sieden erhitzte. Da anderenteils auch durch Extraktion der Caecaldrüsen ein celluloselösendes Enzym nicht gewonnen werden konnte, und, wie ich mich überzeugt habe (vgl. folgende Abhandlung), in den in Betracht kommenden Futtermitteln ein solches nicht vorhanden ist, so dürfte nach meiner Ansicht die celluloselösende Kraft des Caecalsaftes vor allem den in ihm zahlreich lebenden Mikroorganismen zuzuschreiben sein. Daß Mikroorganismen bei der Lösung von Cellulose eine bedeutende Rolle spielen können, geht schon aus den Untersuchungen von Hoppe-Seyler, ${ }^{1}$ ) Tappeiner, $\left.{ }^{2}\right)$ Omelianski ${ }^{3}$ ) u. a. m. hervor; daß sie auch im Darmkanal bei der Lösung der Cellulose beteiligt sein dürften, wird durch das Vorkommen gleicher Arten von Mikroorganismen in den für die Celluloseverdauung in Betracht kommenden Ab-

1) Diese Zeitschrift, Bd. X, S. 409.

$\left.{ }^{2}\right)$ Zeitschrift f. Biol., Bd. XX, S. 52; Bd. XXIV, S. 105.

${ }^{3}$ ) C. R. A., Bd. CXXI, S. 653; Bd. CXXV, S. 970, 1131.

Arch. de scienc. biol., St. Petersburg, Bd. VII, S. 423, Bd. IX, S. 251. 
schnitten des Verdauungsschlauchs ${ }^{1}$ ) (Pansen und Haube der Wiederkäuer, Blinddarm und Colonanfang der anderen Herbivoren und Omnivoren) wahrscheinlich gemacht. Von Ellenberger und von anderer Seite ist diesen Lebewesen, und zwar nicht allein den Bakterien, sondern besonders den Protozoen eine große Bedeutung für die Celluloseverdauung zugeschrieben worden.

Auf die eigenartige Anschauung, daß gewisse Protozoen in den ersten Mägen der Wiederkäuer zum Teil von Cellulose leben, und daß diese Individuen dann im Labmagen durch dessen salzsäurehaltigen Magensaft getötet und verdaut werden und daß dadurch die Cellulose indirekt als Nahrungsmittel der Ruminantien dient, soll hier nicht eingegangen werden.

Um der Frage der Mitwirkung der Mikroorganismen bei der Celluloseverdauung im Caecum näher zu treten, stellte ich mit den Caecalinhalten von Pferd, Schwein und Kaninchen neue Versuche an und wählte folgende Versuchsanordnung:

Zunächst versuchte ich ein von Bakterien und Mikroorganismen freies Filtrat der Caecalinhalte zu gewinnen. $\mathrm{Zu}$ diesem Zwecke wurde der Caecalinhalt zunächst koliert und dann durch Berkefeldfilter filtriert. Infolge der langwierigen Filtration ist es aber nur in wenigen Fällen, und zwar beim Pferde, gelungen, genügende Mengen des Filtrates zu erhalten. Weiterhin wurden gewöhnliche Filtrate unter Anwendung von feinstem Filtrierpapier hergestellt, deren mikroskopische Untersuchung die Abwesenheit von Protozoen ergab. Außerdem wurden die einfachen Kolate, in denen noch sämtliche Mikroorganismen vorhanden waren, für die Versuche verwandt. Von den so gewonnenen Flüssigkeiten gelangte eine abgemessene Menge mit je $1 \mathrm{~g}$ einer aus Heu nach dem Weender Ver-

1) Bundle, Ciliate Infusorien im Caecum des Pferdes, Zeitschrift für wissenschaftl. Zoologie, Bd. LX, S. 284.

Günther, Weitere Beiträge zur Kenntnis des feineren Baues einiger Infusorien im Wiederkäuermagen und im Caecum des Pferdes, Ibid., Bd. LXVII, S. 640 .

Schuberg, Zentralbl. f. Bakteriologie, Bd. III, Nr. 23.

Eberlein, Inaug.-Diss. Leipzig, 1895. 
fahren hergestellten und mit Alkohol und Äther von Farbstoffen befreiten Cellulose auf $65-72$ Stunden bei $39-40^{\circ}$ in den Brütofen. Die Verdauungsflüssigkeit stellte am Ende des Versuches eine dunkle, schmierige, dickliche Flüssigkeit dar. Sie wurde in Schalen gespült und mit je $100 \mathrm{ccm}$ einer 1,25\% \% igen KOH 15 Minuten zum Sieden erhitzt, dann die übrig gebliebene Cellulose auf gewogenen Filtern gesammelt, mit heißem Wasser, verdünnter Essigsäure, Alkohol und Äther gewaschen, bei $105^{\circ}$ getrocknet und dann gewogen. Durch das Kochen in schwach alkalischer Lösung werden einesteils die etwaigen, die Filtration hemmenden Fäulnisprodukte zerstört, während anderenteils die noch vorhandene Cellulose nicht angegriffen wird, so daß eine Beeinträchtigung des Resultates nicht zu befürchten war. Im nachfolgenden sind kurz die diesbezüglichen Versuche aufgeführt.

1. Versuche an Pferden: Pferd I wurde sofort nach der letzten Mahlzeit, die aus Hafer und Heu bestand, getötet. Der Caecuminhalt reagierte alkalisch.

a) $40 \mathrm{ccm}$ Berkefeldfiltrat des Caecalinhaltes gelangten mit $1 \mathrm{~g}$ lufttrockener Gellulose auf 65 Stunden bei $38^{\circ} \mathrm{C}$. in den Brütofen.

Angewandte Cellulose als Trockensubstanz $\quad 0,9290 \mathrm{~g} \quad 0,9290 \mathrm{~g}$

Davon ungelöst zurückgewonnen

\begin{tabular}{cc}
$0,8470>$ & 0,8530 : \\
\hline $0,0820 \mathrm{~g}$ & $0,0760 \mathrm{~g}$
\end{tabular}

$$
\text { Cellulose gelöst in Prozenten } 8,8 \% \quad 8,2 \%
$$

b) $50 \mathrm{ccm}$ Filtrat durch feuchtes Filtrierpapier gelangten mit $1 \mathrm{~g}$ lufttrockener Cellulose auf 65 Stunden in den Thermostaten.

Angewandte Cellulose als Trockensubstanz $0,9290 \mathrm{~g}$

Davon ungelöst zurückgewonnen

$$
\begin{array}{cc}
\text { Gelöste Cellulose } & 0,8330 \\
\cline { 2 - 2 } \text { Cellulose gelöst in Prozenten } & 0,0960 \mathrm{~g} \\
10,3 \%
\end{array}
$$

c) $50 \mathrm{~g}$ Kolat gelangten mit $1 \mathrm{~g}$ lufttrockener Cellulose auf 65 Stunden in den Brütofen.

$$
\begin{array}{rc}
\text { Angewandte Cellulose als Trockensubstanz } & 0,9290 \mathrm{~g} \\
\text { Davon ungelöst zurückgewonnen } & 0,6940 \mathrm{~g} \\
\cline { 2 - 2 } \text { Gelöste Cellulose } & 0,2350 \mathrm{~g} \\
\text { Cellulose gelöst in Prozenten } & 25,3 \%
\end{array}
$$

Pferd II, mit Hafer und Heu gefüttert, wurde 2 Stunden nach der letzten Mahlzeit getötet. Caecuminhalt reagiert alkalisch und ist von bräunlicher Farbe.

a) $100 \mathrm{ccm}$ Papierfiltrat gelangen mit $1 \mathrm{~g}$ lufttrockener Cellulose auf 72 Stunden bei $38^{\circ}$ in den Thermostaten. 
Angewandte Cellulose als Trockensubstanz $\quad 0,9105 \mathrm{~g} \quad 0,9105 \mathrm{~g}$

Davon ungelöst zurückgewonnen

$\begin{array}{rcc}\text { Gelöste Cellulose } & 0,2377 \mathrm{~g} & 0,2239 \mathrm{~g} \\ \text { Cellulose gelöst in Prozenten } & 26,1 \% & 24,6 \%\end{array}$

b) $100 \mathrm{ccm}$ Kolat gelangten mit $1 \mathrm{~g}$ lufttrockener Cellulose auf 72 Stunden in den Thermostaten.

\begin{tabular}{ccc} 
Angewandte Cellulose als Trockensubstanz & $0,9105 \mathrm{~g}$ & $0,9105 \mathrm{~g}$ \\
Davon ungelöst zurückgewonnen & $0,6192 \mathrm{y}$ & 0,6595 \\
\cline { 2 - 3 } Gelöste Cellulose & $0,2913 \mathrm{~g}$ & $0,2510 \mathrm{~g}$ \\
Gelöste Cellulose in Prozenten & $32,0 \%$ & $27,6 \%$
\end{tabular}

Die vorstehenden Versuchsergebnisse zeigen deutlich, daß der normale Caecalinhalt in seiner celluloselösenden Wirkung durch die Filtration erheblich beeinträchtigt wird. In allen Fällen war die Verdauung der Cellulose bei den nur durch Kolieren von groben Beimengungen befreiten Flüssigkeiten am größten. Bei Pferd I war die Celluloseverdauung des Kolates sogar mehr als doppelt so groß als die des durch Papier filtrierten Inhaltes. Den niedrigsten Wert zeigt das mikroorganismenfreie Berkefeldfiltrat. Der Unterschied zwischen diesem und dem Papierfiltrat ist nur unbedeutend. Da infolge der schleimigen Beschaffenheit und ihres Gehaltes an Kolloiden die zu filtrierende Flüssigkeit die Poren des Filtrierpapiers sehr rasch verstopft, was sich schon dadurch anzeigt, daß die Filtration nur sehr langsam vonstatten geht, werden auf dem Filter die Mikroorganismen, vor allem die Protozoen, zurückgehalten oder gelangen wenigstens nur in verschwindender Anzahl in das Filtrat. Ich glaube infolgedessen aus den vorliegenden Resultaten zu dem Schlusse berechtigt zu sein, daß vor allem Mikroorganismen bei der Lösung der Cellulose im Caecum eine wichtige Rolle spielen, und daß besonders das Vorhandensein von Protozoen für die Beträchtlichkeit der Celluloseverdauung von Wichtigkeit ist. Beim einfachen Kolieren des Caecuminhaltes durch Tücher gelangen diese durch die weiten Maschen des Koliertuches in das Kolat; dieses entfaltet dann die größte verdauende Wirkung auf die Cellulose. Besonders instruktiv scheint mir ein weiterer Versuch zu sein, den ich mit der Caecalflüssigkeit eines dritten Pferdes anstellte. Dieses war von mir mit Mais für andere $Z$ wecke gefüttert worden und wurde 9 Stunden 
nach der letzten Mahlzeit getötet; sein Caecalinhalt reagierte sauer, zeigte also eine abnorme, nur bei sehr amylaceenreicher Nahrung auftretende Reaktion (Ellenberger, ${ }^{1}$ ) Scheunert und Grimmer ${ }^{2}$ ).

$50 \mathrm{ccm}$ Berkefeldfiltrat verdauten in 0,2\% iger Sodalösung von $1 \mathrm{~g}$ vorgelegter Cellulose in 54 Stunden nur 2,3\% und $50 \mathrm{ccm}$ Papierfiltrat unter denselben Bedingungen nur 6,6\%. Durch die saure Reaktion, die das Gedeihen gewisser Mikroorganismen hindert bezw. diesen die Lebensbedingungen entzieht, wird also eine Minderung der cytohydrolytischen Wirkung des Caecalinhaltes verursacht.

2. Versuche am Schwein: Der Caecalinhalt eines Schweines, welches, wie die Untersuchung des Magendarminhaltes ergab, mit Mehl und Hafer gefüttert worden war, wurde koliert und durch Papier filtriert. $\mathrm{Da}$ es infolge der Beschaffenheit des Caecuminhaltes nicht möglich war, ein Berkefeldfiltrat $\mathrm{zu}$ erhalten, mußte auf dieses verzichtet werden.

a) $25 \mathrm{ccm}$ Papierfiltrat gelangten mit $1 \mathrm{~g}$ lufttrockener Cellulose auf 72 Stunden in den Brutofen und wurden dann, wie oben geschildert, weiter behandelt.

\begin{tabular}{cc} 
Angewandte Cellulose als Trockensubstanz & $0,9290 \mathrm{~g}$ \\
Davon ungelöst zurückgewonnen & 0,8475 , \\
\cline { 2 - 2 } Gelöste Cellulose & $0,0815 \mathrm{~g}$ \\
In Prozenten & $8,8 \%$
\end{tabular}

b) $25 \mathrm{ccm}$ Kolat wurden unter denselben Bedingungen behandelt. Angewandte Cellulose als Trockensubstanz $\quad 0,9290 \mathrm{~g} \quad 0,9290 \mathrm{~g} \quad 0,9290 \mathrm{~g}$ Davon ungelöst zurückgewonnen

Gelöste Cellulose $\frac{0,7980 \times 0,7310 \mathrm{~g} 0,1560 \mathrm{~g} \quad 0,1200 \mathrm{~g}}{0,130,0,8090 \mathrm{~g}}$ In Prozenten $\quad 14,1 \% \quad 16,8 \% \quad 12,9 \%$

Auch beim Schwein hatte also der filtrierte Caecalinhalt nur etwa die Hälfte der Cellulose gelöst, die der kolierte unter gleichen Bedingungen zu lösen imstande war.

3. Versuche am Kaninchen: Auch bei diesen Tieren war es nicht möglich, ein Berkefeldfiltrat zu erhalten. Es machte sich sogar nötig, den dicken Caecuminhalt mit dem gleichen Volumen Wasser zu verdünnen, um eine Filtration zu ermöglichen.

I. Von zwei Kaninchen, die mit Heu, Rüben und Kraut gefüttert worden waren, wurden $50 \mathrm{ccm}$ Filtrat aus der Caecalflüssigkeit erhalten.

1) Archiv f. wissenschaftl. u. prakt. Tierheilkunde, Bd. XIV, S. 129.

2) Diese Zeitschrift, Bd. XLVII, S. 98. 
Je $25 \mathrm{ccm}$ hiervon gelangten während 68 Stunden mit $1 \mathrm{~g}$ lufttrockener Cellulose in den Brütofen.

\begin{tabular}{rcc} 
Angewandte Cellulose als Trockensubstanz & $0,9290 \mathrm{~g}$ & $0,9290 \mathrm{~g}$ \\
Davon ungelöst zurückgewonnen & $0,8518>$ & 0,8582 \\
\cline { 2 - 3 } Verschwundene Cellulose & $0,0772 \mathrm{~g}$ & $0,0708 \mathrm{~g}$ \\
In Prozenten & $8,3 \%$ & $7,6 \%$
\end{tabular}

II. Von einem Kaninchen, welches mit Hafer gefüttert worden war, gelangten Filtrate und Kolate der 1:1 mit Wasser verdünnten Caecalflüssigkeit mit je $1 \mathrm{~g}$ lufttrockener Cellulose auf 72 Stunden in den Brutofen.

a) Filtrat: Angewandte Cellulose als Trockensubstanz $0,9290 \mathrm{~g}$ Davon ungelöst zurückgewonnen $\frac{0,8590 \text { 工 }}{0,0700 \mathrm{~g}}$ $\begin{array}{rr}\text { Verschwundene Cellulose } & 0,0700 \\ \text { In Prozenten } & \mathbf{7 , 5} \%\end{array}$

b) Kolat: Angewandte Cellulose als Trockensubstanz $0,9290 \mathrm{~g} \quad 0,9290 \mathrm{~g}$ Davon ungelöst zurückgewonnen Verschwundene Cellulose $\frac{0,7765>0,7590 \text { > }}{0,1525 \mathrm{~g} \quad 0,1700 \mathrm{~g}}$ In Prozenten 16,4\% 18,3\%

Auch beim Kaninchen wird also die Fähigkeit des Caecalsaftes durch Filtration desselben durch feinstes Filtrierpapier um die Hälfte verringert.

Die im vorstehenden geschilderten Versuche zeigen, daß die Caecalflüssigkeiten von Pferd, Schwein und Kaninchen eine celluloselösende Wirkung auszuüben vermögen und daß diese durch die Filtration der Flüssigkeiten durch Bakterienfilter oder feinstes Filtrierpapier ganz erheblich beeinträchtigt wird. Eine Erklärung dieser merkwürdigen Erscheinung dürfte dadurch gegeben sein, daß die in dem Blinddarm vorhandenen Mikroorganismen hauptsächlich die Lösung der Cellulose bewirken, und daß die Caecalflüssigkeit in ihrer celluloselösenden Wirkung erheblich geschwächt wird, wenn diese Mikroorganismen aus ihr entfernt werden (durch Filtration oder Abtötung). Die Berechtigung zu dieser Annahme ist um so größer, als, wie eingangs erwähnt, weder aus der Caecalflüssigkeit noch aus den Extrakten der Caecalschleimhaut ein cytohydrolytisches Enzym gewonnen werden konnte, und da festgestellt worden ist, daß die gekochte Caecalflüssigkeit Cellulose nicht mehr zu lösen vermochte. 
Auffallend bleibt immerhin, daB das Berkefeld filtrat bei alkalischer Reaktion des Caecalinhaltes Cellulose löst, wenn auch erheblich weniger als das an Mikroorganismen reiche Kolat. Da die Berkefeldfiltrate bekanntlich Mikroorganismen nicht enthalten, muß man annehmen, daß Körper durch das Filter hindurch gehen, die Cellulose zu lösen vermögen. $\mathrm{Da}$ weiterhin nachträglich von außen voraussichtlich keine Mikroorganismen in die Verdauungsgefäße gelangt sind (Verschluß derselben durch Watte usw.), könnte es sich hier um Sekrete der Mikroorganismen handeln, die enzymatisch auf Cellulose zu wirken vermögen.

Sollte dies der Fall sein, so bleibt es immerhin auffällig, daß diese Stoffe bisher nicht isoliert werden konnten. Wichtig für die Lösung der Frage nach der Ursache der Celluloselösung erscheint die Tatsache, $\mathrm{da}$ \ die Caecalflüssigkeit in ihrer Fähigkeit, Cellulose zu lösen, bedeutend beeinträchtigt wird, wenn ihre Reaktion (infolge von sehr stärkereicher Kost) sauer ist. Die saure Reaktion hindert die Lösung der Cellulose, vielleicht dadurch, daß die dabei beteiligten Mikroorganismen unter diesen Verhältnissen nicht gedeihen, sich nicht fortpflanzen oder die von ihnen produzierten Enzyme durch die Säure wirkungslos oder zerstört werden.

Es mag hier auch noch darauf hingewiesen werden, daß bei den früheren, in unserem Institute (mit Kolaten und durch grobes Filtrierpapier unter häufigem Filterwechsel gewonnenen Mikroorganismen enthaltenden Filtraten) ausgeführten Versuchen bedeutend mehr Cellulose 'gelöst wurde als bei den neueren Versuchen. Dies erklärt sich daraus, daß damals größere Mengen Caecalsaft und zum Teil anders hergestellte, zartere, weniger widerstandsfähige Cellulose angewandt worden ist. Vor allem haben die früheren Versuche ergeben, daß die Menge der gelösten Cellulose von der Dauer des Versuches, also von der Zeit abhängt, die die cellulosehaltigen Nahrungsmittel in den betreffenden Darmabschnitten verweilen.

Zur Untersuchung auf die in der Caecalflüssigkeit enthaltenen Enzyme wurden Caecalflüssigkeiten von Pferden verwendet. Die Tiere wurden vorher mit Hafer gefüttert (Heu ist wegen der darin enthaltenen, die Versuche störenden Farbstoffe zu vermeiden) und dann einige Zeit nach der letzten Mahlzeit getötet. Der Caecuminhalt wurde zunächst koliert und dann filtriert. Die Farbe des Filtrates war zunächst hellbraun, veränderte sich aber bald, indem sie nach einigen Stunden in dunkelbraun umschlug. Die Reaktion war stets alkalisch. Zucker und Pepton waren nie deutlich nachzuweisen, ersterer quantitativ nie bestimmbar.

Auf diastatische Enzyme wurde in der Weise geprüft, daß $25 \mathrm{ccm}$ Caecalflüssigkeit mit Stärkekleister (bereitet aus

Hoppe-Seyler's Zeitschrift f. physiol. Chemie. XIVIII. 
$1 \mathrm{~g}$ Kartoffelstärke $+30 \mathrm{ccm} \mathrm{H}_{2} \mathrm{O}$ ) auf 6 Stunden bei $39-40^{\circ}$ in den Thermostaten eingestellt wurden. Nach Ablauf dieser Zeit wurden die die Zuckerbestimmung störenden Eiweißkörper mit Salzsäure und Phosphorwolframsäure gefällt und nach dem Abfiltrieren und Alkalisieren des Filtrates die Zuckerbestimmung ausgeführt.

Je $25 \mathrm{ccm}$ von 3 verschiedenen Caecalflüssigkeiten hatten in 6 Stunden gebildet: $1.0,113 \mathrm{~g}, 2.0,166 \mathrm{~g}, 3.0,144 \mathrm{~g}$ Zucker (berechnet als Dextrose).

Die Anwesenheit eines diastatischen Enzyms wird durch diesen und meine andern Versuche von neuem bestätigt.

Die Prüfung auf ein proteolytisches Enzym erfolgte in der Weise, daß die Einwirkung der Caecalflüssigkeit auf Fibrin und koaguliertes Hühnereiweiß beobachtet wurde. Nach kürzerem oder längerem (8-24 Stunden) Aufenthalt im Brütofen trat stets gänzliche oder teilweise Lösung der vorgelegten Eiweißkörper ein, sodaß auf die Anwesenheit eines proteolytischen Enzyms ebenfalls geschlossen werden muß.

Auf ein Milchsäureenzym wurde in der Weise geprüft, daß $25 \mathrm{ccm}$ der filtrierten Caecalflüssigkeit mit $5 \mathrm{~g}$ Glukose auf 24 Stunden in den Thermostaten eingestellt wurden; ebenso gelangten zur Untersuchung auf ein invertierendes Enzym $5 \mathrm{~g}$ Rohrzucker mit $25 \mathrm{ccm}$ Caecalflüssigkeit auf 5 Stunden in den Brütofen. In allen Fällen konnte die Anwesenheit eines Milchsäure bildenden und eines Rohrzucker invertierenden Enzyms festgestellt werden. In völliger Übereinstimmung mit den früheren Versuchsergebnissen von Ellenberger und $\mathrm{Hofmeis}$ ter konnte nach dem vorstehenden ein diastatisches, proteolytisches, milchsäurebildendes und invertierendes Enzym im Caecalsaft des Pferdes nachgewiesen werden.

\section{Untersuchungen über den Enzymgehalt der Caecalschleimhaut.}

Der Enzymgehalt der Caecalschleimhaut ist schon bei früheren Arbeiten in unserem Institute Gegenstand der Untersuchung gewesen. Es wurde damals aber nur die Caecalschleimhaut einer Tierart, des Pferdes, und zwar durch Herstellung 
verschiedener Extrakte derselben, genauer untersucht. Im folgenden sollen die Resultate meiner Versuche, die sich auf den Enzymgehalt der Caecalschleimhaut von Pferd, Kaninchen und Schwein erstreckten, mitgeteilt werden. Bei diesen sind außer Extrakten auch die Preßsäfte der frischen Schleimhäute verwendet worden.

Zur Methodik ist folgendes zu bemerken: Die Caeca wurden den Tieren lebenswarm entnommen, die Schleimhaut rom Inhalte sorgfältig befreit und, falls die Tötung der Tiere nicht im Institut selbst geschah, während des Transportes in Flaschen mit physiologischer Kochsalzlösung eingelegt. Dann wurde bei den Caeca des Pferdes die Schleimhaut abpräpariert, während es bei den Caeca der anderen Tiere aus praktischen Gründen unterblieb. Zur Entfernung etwa imbibierter Enzyme wurden dann die Schleimhäute resp. die Caecalwände während 24 Stunden in fließendem Wasser gewaschen und der aus den Drüsen und dem Oberflächenepithel austretende Schleim von Zeit zu Zeit durch Abstreifen entfernt. Nach dieser Vorbereitung wurden die Schleimhäute und Caeca zu den Extrakten und Preßsäften verarbeitet. Im allgemeinen wurde hierbei das Material in zwei Portionen, die Schleimhaut des blinden Endabschnittes und die des Caecumkörpers oder des Caecumkopfes, getrennt. Die Schleimhaut der Caecumspitze besitzt beim Pferd zahlreiche dicht gelegene, große Lymphknötchen, die eine förmliche Platte bilden, welche nach Ellenberger (Arch. für Anat. u. Physiol., 1906, S. 139) als Anologon des Processus vermiformis anzusehen ist. Beim Kaninchen ist dies noch ausgesprochener, sodaß man bei diesem Tiere von einem echten Wurmfortsatz mit einer total cytoblastischen Schleimhaut sprechen muß. Beim Schwein ist weder ein Wurmfortsatz noch eine größere Anhäufung von cytoblastischem Gewebe im blinden Ende des Caecums vorhanden, hingegen finden sich in dem Anfangsteile des Caecumkörpers und vor allem an der lleocaecalklappe Follikelplatten. Zur Extraktion und Gewinnung von Preßsäften verwandte ich stets einesteils die Schleimhaut des Caecumkörpers und -kopfes beim Pferd und den Caecumkörper beim Schwein und Kaninchen, anderenteils die die Lymphknötchen enthaltende Schleimhautportion der Caecumspitze des Pferdes, das blinde Caecumende des Schweines und die cytoblastische Schleimhaut des Wurmfortsatzes vom Kaninchen. Zur leichteren Orientierung sind im folgenden die zuerst erwähnten Extrakte und Preßsäfte als solche des Körpers und die zuletzt erwähnten als solche der Spitze bezeichnet worden.

Die betreffenden Blinddarmteile wurden fein zerhackt und zur Extraktion beim Pferd mit der doppelten Gewichtsmenge 1\% iger Sodalösung und Chloroformwasser, beim Pferd, Schwein und Kaninchen mit der anderthalbfachen Menge Glycerin, welches im Verhältnis 2 Glycerin:1 Wasser verdünnt war, angesetzt. Die Dauer der Extraktion 
betrug mit Sodalösung und Chloroformwasser nur 24 Stunden, mit Glycerin wurde sie auf 6 Tage und mehr ausgedehnt. Die Extrakte waren nach der Filtration leicht gelblich bis rötlich gefärbte Flüssigkeiten von mehr oder weniger schleimiger Beschaffenheit, je nachdem sie aus dem Körper oder der Spitze des Caecums hergestellt worden waren. In keinem Falle zeigten sie eine Pepton- oder Zuckerreaktion.

Zur Herstellung der Preßsäfte wurden die fein zerhackten Blinddarmteile mit Quarzsand innig verrieben und dann bei 300 Atmosphären Druck ausgepreßt. Nach dem Filtrieren waren die Preßsäfte leicht rötlich gefärbte, meist dünnflüssige und bei längerer Aufbewahrung sich trübende Flüssigkeiten, die weder Zucker noch Pepton enthielten.

a) Prüfung auf proteolytisches Enzym. Die Prüfung wurde derart vorgenommen, daß 2, 3 oder 2,5 ccm der Extrakte und Preßsäfte auf Fibrin, koaguliertes Hühnereiweiß und Met tsche Röhrchen, die mit koaguliertem Ochsenblutserum, welches mit $50 \%$ Wasser verdünnt war, gefüllt waren, während 6 und 24 Stunden im Brütofen bei $40^{\circ}$ einwirkten. Zur Verdünnung wurden

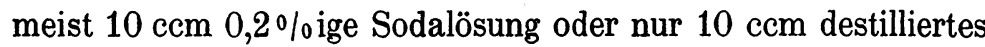
Wasser gewählt, so daß die Einwirkung bei neutraler und alkalischer Reaktion, letztere entspricht der normalen Reaktion des Caecalinhalts, studiert werden konnte. In einigen Fällen wurde auch durch Zusatz von $5 \mathrm{ccm} 0,2 \%$ iger Salzsäure eine saure Reaktion des Verdauungsgemisches hergestellt. Nach Ablauf der Digestionszeit wurde mit dem Auge festgestellt, ob eine Verdauung der vorgelegten Eiweißkörper eingetreten war, und eine Prüfung der Filtrate der Verdauungsflüssigkeiten auf Pepton vorgenommen.

Die zahlreichen, mit den Extrakten des Caecums vom Pferde unter den mannigfaltigsten Variationen angestellten Versuche einzeln hier aufzuführen, würde zu weit führen; bezüglich ihrer Resultate sei erwähnt, daß ich nur in zwei Fällen eine proteolytische Wirkung eines Sodaextraktes und eines Glycerinextraktes bei alkalischer Reaktion feststellen konnte. Andere derartige Extrakte sowie die Chloroformwasserauszüge und die Preßsäfte hatten hingegen niemals, weder eine Lösung noch eine Peptonisierung der vorgelegten Eiweißkörper bewirkt. Gerade diese Unstimmigkeit war die Veranlassung, die Versuche in möglichst großer Anzahl unter allen möglichen Variationen der Versuchsbedingungen anzustellen. Außerdem versuchte ich eine Aufklärung dadurch zu erlangen, 
daß ich mit der Caecalschleimhaut des Pferdes einen Autodigestionsversuch unternahm.

Die wie oben geschildert präparierte und vorbereitete Schleimhautportion von Körper und Follikelplatte wurden fein zerhackt, mit Quarzsand innig zerrieben und in neutraler Lösung unter Toluol 8 Wochen im Brutschrank bei $40^{\circ}$ sich selbst überlassen. Das Toluol wurde, wenn eine Verminderung bemerkt wurde, ersetzt. Nach dieser Zeit wurde abfiltriert; dabei wurden klare dünnflüssige, dunkelfarbige Filtrate von neutraler Reaktion erhalten, die sich durch ihr Aussehen nicht voneinander unterschieden. Auch in bezug auf ihre chemischen Reaktionen zeigten sie sich völlig gleich, sie zeigten keine Tryptophanreaktion, enthielten kein Pepton, gaben eine schwach positive Reaktion mit Millons Reagens und eine ebenfalls geringe Fällung mit Phosphorwolframsäure. Zur Prüfung auf proteolytische Wirkung gelangten je $2,5 \mathrm{ccm}$ mit je $25 \mathrm{ccm}$ Wasser, $0,25 \%$ ige $\mathrm{HCl}$ - und $0,2 \%$ ige $\mathrm{Na}_{2} \mathrm{CO}_{8}$-Lösung mit je $1 \mathrm{~g}$ Würfeln aus koaguliertem Hühnereiweiß in den Brütofen. Nach 21 Stunden war noch keine Peptonisierung des Eiweißes eingetreten.

Auch auf Grund dieses Versuches erscheint der Schluß berechtigt, daß von den Caecaldrüsen des Pferdes ein proteolytisches Enzym nicht produziert wird, und daß die gegenteiligen Resultate auf Zufälligkeiten (Art und Weise und Zeit des Auswaschens usw.) beruhen.

Diese Anschauung wird noch weiter durch die Ergebnisse der Untersuchungen von Preßsäften und Extrakten der Caeca vom Schwein und Kaninchen wesentlich unterstützt.

Die Versuchsanordnung war folgende:

Von den auf die oben geschilderte Weise (aus Körper und Spitze) hergestellten Extrakten und Preßsaften wurden je $3 \mathrm{ccm}$ mit $10 \mathrm{ccm}$ einer $0,2 \%$ igen Sodalösung und $1 \mathrm{~g}$ Würfeln aus koaguliertem Hühnereiweiß bei $40^{\circ}$ in den Thermostaten eingestellt. Nach 24 Stunden, während welcher Zeit öfter umgeschüttelt worden war, wurde der Versuch unterbrochen, geprüft, ob schon der Augenschein eine Lösung von Eiweißwürrfeln ergab, dann abfiltriert und mit dem Filtrat die Peptonreaktion angestellt.

Auch hier konnte weder in den Glycerinextrakten noch in den Preßsäften aus Caecumkörper und Follikelplatte (Proc. vermiformis) die Anwesenheit eines proteolytischen Enzyms nachgewiesen werden, da in allen Fällen die Peptonreaktion negativ war.

Ein proteolytisches Enzym scheint demnach von den Caecaldrüsen der untersuchten Tiere nicht produziert zu werden. 
b) Diastatisches, Milchsäure- und andere Enzyme. Die Untersuchung auf diese Enzyme erfolgte zuerst in der Weise, daß einige Kubikzentimeter der Extrakte und Preßsäfte auf $1 \mathrm{~g}$ verkleisterte Stärke einwirken gelassen wurden. Es stellte sich hierbei aber bald heraus, daß einesteils die diastatische Wirkung zu gering war, um in der zu einem einwandfreien Versuche nötigen kurzen Zeit (10 Stunden wurden nicht überschritten) die vorgelegte Stärke bis zu reduzierenden Produkten abzubauen. Auf längere Zeit, etwa auf 24 Stunden, einen Versuch auszudehnen, erschien unzweckmäßig, da dann leicht von außen in das Digestionsgefä $ß$ hineingelangte Keime und Fermente einen Abbau der Stärke herbeiführen können, wie das von Goldschmidt bei seinen Untersuchungen über die diastatische Wirkung des Speichels in unserem Institut nachgewiesen worden ist. Es wurden infolgedessen nur wenige Kubikzentimeter eines ganz verdünnten und zwar 1\% $1 \%$ igen Stärkekleisters vorgelegt. Die Prüfung auf die durch etwaige diastatische Wirkung entstandenen Spaltungsprodukte der Stärke erfolgte außer mit Fehlingscher Lösung auch noch durch Anstellung der Jodreaktion. Ebenso wurde in allen Fällen auf das Vorhandensein von Milchsäure vermittelst des Uffelmannschen Reagenses geprüft. Sämtliche Versuche wurden bei neutraler Reaktion vorgenommen. Die Versuchsergebnisse sind in der folgenden Übersicht tabellarisch geordnet.

Aus der nebenstehenden Tabelle geht mit Sicherheit hervor, daß die Caecaldrüsen ein amylolytisches Enzym enthalten. Allerdings scheint dasselbe teilweise nur in schwacher Wirksamkeit vorhanden zu sein, so daß es z. B. in den Glycerinextrakten des Caecums des Kaninchens nicht mit Sicherheit nachgewiesen werden konnte. Die geringe saccharizierende Wirkung der Extrakte und Preßsäfte ließ die Vermutung aufkommen, daß es sich hier nicht um ein in den Caecaldrüsen produziertes Enzym, sondern um die amylolytische Wirksamkeit handele, die fast alle Körperextrakte auszuüben imstande sind. ${ }^{1}$ )

1) Ellenberger, Die Verbreitung des saccharifizierenden Fermentes im Pferdekörper, Archiv f. wissenschaftl. u. prakt. Tierheilkunde, Bd. VIII, S. 91. 
Es sind daher Kontrollversuche mit Extrakten aus der Caecalmuskulatur, der Skelettmuskulatur u. a. m. angesetzt worden. Hierbei hat sich ergeben, daß die amylolytische Wirkung derartiger Extrakte viel geringer als die der Extrakte und Preßsäfte der Caecalschleimhaut ist; denn in keinem Falle konnte schon in 6 Stunden eine deutliche Verzuckerung des vorgelegten Kleisters festgestellt werden. Die Extrakte der gekochten Caecalschleimhaut waren völlig wirkungslos.

\begin{tabular}{|c|c|c|c|c|c|c|c|c|}
\hline Tierart & \multicolumn{2}{|c|}{$\begin{array}{c}\text { Art der } \\
\text { untersuchten } \\
\text { Flüssigkeit }\end{array}$} & $\begin{array}{l}\text { Ex- } \\
\text { trakt } \\
\mathrm{ccm}\end{array}$ & $\begin{array}{l}\text { Di- } \\
\text { gestions- } \\
\text { dauer } \\
\text { in } \\
\text { Stunden }\end{array}$ & $\begin{array}{c}\text { Anzahl } \\
\text { des vor- } \\
\text { gelegten } \\
\text { Stärke- } \\
\text { kleisters } \\
\text { ccm }\end{array}$ & $\begin{array}{c}\text { Jod- } \\
\text { reaktion }\end{array}$ & $\mid \begin{array}{c}\text { Fehling- } \\
\text { sche } \\
\text { Lösung }\end{array}$ & $\begin{array}{l}\text { Milch- } \\
\text { säure }\end{array}$ \\
\hline \multirow{4}{*}{ Pferd } & \multirow{2}{*}{$\begin{array}{l}\text { Chloro- } \\
\text { form- } \\
\text { extrakt }\end{array}$} & Körper & 2 & 5 & 10 & rot & $\begin{array}{c}\text { wird nicht } \\
\text { reduziert }\end{array}$ & $\begin{array}{c}\text { nicht vor- } \\
\text { handen }\end{array}$ \\
\hline & & Spitze & 2 & 5 & 10 & $\begin{array}{l}\text { Achroo- } \\
\text { dextrin? } \\
\text { farblos }\end{array}$ & $\begin{array}{l}\text { wird nicht } \\
\text { reduziert }\end{array}$ & $\begin{array}{l}\text { nicht vor- } \\
\text { handen }\end{array}$ \\
\hline & \multirow{2}{*}{ Preßsaft } & Körper & 2 & 6 & 20 & rot & $\begin{array}{c}\text { wird } \\
\text { reduziert }\end{array}$ & $\begin{array}{c}\text { vor- } \\
\text { handen }\end{array}$ \\
\hline & & Spitze & 2 & 6 & 20 & rot & $\begin{array}{c}\text { wird } \\
\text { reduziert }\end{array}$ & $\begin{array}{c}\text { vor- } \\
\text { handen }\end{array}$ \\
\hline \multirow{4}{*}{ Sichwein } & \multirow{2}{*}{$\begin{array}{c}\text { Glycerin- } \\
\text { extrakt }\end{array}$} & Körper & 3 & 6 & 25 & rot & $\begin{array}{c}\text { wird } \\
\text { reduziert }\end{array}$ & $\begin{array}{c}\text { vor- } \\
\text { handen }\end{array}$ \\
\hline & & Spitze & 3 & 6 & 25 & rot & $\begin{array}{c}\text { wird } \\
\text { reduziert }\end{array}$ & $\begin{array}{c}\text { vor- } \\
\text { handen }\end{array}$ \\
\hline & \multirow{2}{*}{ Preßsaft } & Körper & 3 & 6 & 25 & blau & $\begin{array}{c}\text { wird } \\
\text { reduziert }\end{array}$ & $\begin{array}{c}\text { vor- } \\
\text { handen }\end{array}$ \\
\hline & & Spitze & 3 & 6 & 25 & blau & $\begin{array}{c}\text { wird } \\
\text { reduziert }\end{array}$ & $\begin{array}{c}\text { vor- } \\
\text { handen }\end{array}$ \\
\hline \multirow{4}{*}{ Kæninchen } & \multirow{2}{*}{$\begin{array}{l}\text { Glycerin- } \\
\text { extrakt }\end{array}$} & Körper & 3 & 6 & 25 & blau & $\begin{array}{c}\text { wird nicht } \\
\text { reduziert }\end{array}$ & $\begin{array}{c}\text { vor- } \\
\text { handen }\end{array}$ \\
\hline & & Spitze & 3 & 6 & 25 & blau & \begin{tabular}{|c} 
wird nicht \\
reduziert
\end{tabular} \mid & $\begin{array}{c}\text { vor- } \\
\text { handen }\end{array}$ \\
\hline & \multirow{2}{*}{ Preßsaft } & Körper & 3 & 6 & 25 & rot & $\begin{array}{c}\text { wird } \\
\text { reduziert }\end{array}$ & $\begin{array}{c}\text { vor- } \\
\text { handen }\end{array}$ \\
\hline & & Spitze & 3 & 6 & 25 & rot & $\begin{array}{c}\text { wird } \\
\text { reduziert }\end{array}$ & $\begin{array}{c}\text { vor- } \\
\text { handen }\end{array}$ \\
\hline
\end{tabular}

Ebenso wie das Vorhandensein eines amylolytischen Enzyms in dem Caecalsekret muß auch das eines Milchsäureenzyms angenommen werden, da wir 
in allen in Betracht kommenden Fällen das Auftreten freier Milchsäure feststellen konnten, wie die umstehende Tabelle zeigt. Die bei der Autolyse der Caecalschleimhaut des Pferdes gewonnenen Extrakte zeigten ebenfalls eine amylolytische Wirkung, indem sie bei der Einwirkung auf Stärkekleister eine Umwandlung dieses in reduzierende Substanzen bewirkten; ebenso war auch in ihnen ein Milchsäure bildendes Enzym vorhanden.

Im Anschluß an diese Versuche sind auch Untersuchungen der Extrakte und Preßsäfte auf ihren Gehalt an Erepsin und Enterokinase angestellt worden.

Zur Prüfung auf Erepsin wurden die Extrakte und Preßsäfte, die kein Eiweiß verdauten, mit stark verdünnter Peptonlösung, die nach Co hnheim ${ }^{1}$ ) hergestellt worden war, auf 24 Stunden in den Brütofen eingestellt. Dann wurden die Eiweißkörper mit $\mathrm{Na}_{2} \mathrm{SO}_{4}$ und $\mathrm{CH}_{8} \mathrm{COOH}$ in der Siedehitze gefällt und im Filtrat die Biuretreaktion ausgeführt. In allen Fällen trat eine starke Peptonreaktion auf, Erepsin war demnach nicht zugegen.

Zur Prüfung auf Enterokinase wurden Mettsche Röhrchen verwendet, die nach dem Vorschlag von Prym $\left.{ }^{2}\right)$ mit Gelatine $\left(100 \mathrm{H}_{2} 0\right.$, $5 \mathrm{~g}$ Gelatine, $5 \mathrm{ccm} 5 \%$ igiges Formalin, 1 Tropfen Methylenblau) gefüllt waren. Ferner wurde ein Extrakt vom Pankreas des Pferdes derart bergestellt, daß die Drüse lebenswarm unter Glycerin gebracht, daselbst zerkleinert und dann 8 Tage der Extraktion überlassen wurde. Der Extrakt zeigte nach 6 Stunden noch keine Einwirkung auf die Mettschen Röhrchen, enthielt also das Trypsin nur in seiner unwirksamen Vorstufe. $2 \mathrm{ccm}$ Pankreasextrakt und $2 \mathrm{ccm}$ Extrakt resp. Preßsaft der Caecalschleimhaut des Pferdes gelangten dann verdünnt mit $5 \mathrm{ccm}$ Wasser mit den Mettschen Röhrchen auf 6 Stunden in den Brütofen.

Nach dieser Zeit war eine Auflösung der Gelatine noch nicht erfolgt. Wurde der Aufenthalt im Brütofen auf 21 Stunden ausgedehnt, so war allerdings eine teilweise Auflösung der Gelatine eingetreten und zwar war von den Extrakten und Preßsäften der die Follikelplatten enthaltenden Teile der Caecalschleimhaut am meisten Gelatine gelöst worden. Bei 21 Stunden Verdauungszeit war aber auch bei den Kontrollversuchen ohne Zusatz von Caecalextrakten eine Lösung der Gelatine eingetreten, was nicht verwunderlich erscheint, da infolge der langen Versuchsdauer wohl infolge äußerer Einflüsse eine Überführung

1) Diese Zeitschrift, Bd. XXXIII, S. 451.

2) Pflügers Archiv, Bd. CIV, S. 440. 
des Protrypsins in Trypsin stattgefunden haben dürfte. Dieses Resultat dürfte also wohl kaum einwandfrei sein und es findet auch nur deshalb hier eine Erwähnung, weil nach der Anschauung von Delezenne ${ }^{1}$ ) gerade die cytoblastischen Gewebe der Darmschleimhaut die Träger der Enterokinase sein sollen. Diese Behauptung ist nicht obne Widerspruch von anderer Seite geblieben; stellt man sich aber trotzdem auf Delezennes Standpunkt, so ist es nicht unmöglich, daß durch das bei den im vorstehenden geschilderten Versuchen zur Entfernung imbibierter Enzyme unbedingt erforderliche lange Auswaschen und Wässern der Schleimhäute gerade die gegen derartige Einflüsse sehr empfindlichen Lympfknötchen zerstört, mazeriert und entfernt worden sind. Nach obigem Versuch scheint es zum mindesten sehr unwahrscheinlich zu sein, daß Enterokinase in den cytoblastischen Geweben" des Caecums enthalten ist.

Schließlich sei noch erwähnt, daß übereinstimmend mit den früheren in unserem Institute ausgeführten Untersuchungen die Anwesenheit eines lipolytischen Enzyms in der Caecalschleimhaut nicht festgestellt werden konnte.

Aus den Ergebnissen der im vorstehenden kurz skizzierten Versuche folgt, daß die aus dem alkalischen Caecuminhalt des Pferdes, des Schweines und des Kaninchens zu gewinnende Flüssigkeit Cellulose in nicht unerheblicher Menge löst. Beim Kochen büßt sie diese Fähigkeit ein, doch ist durch Fällen mit Alkohol aus ihr ein Cellulose lösender Niederschlag nicht zu gewinnen. Die Menge der gelösten Cellulose ist abhängig vom Reichtum an Mikroorganismen, von der Dauer der Einwirkung und von der Quantität der $z u$ den Digestionsversuchen benutzten Caecalflüssigkeit, sowie von der Art und der Herstellung der Cellulose. Die an Mikroorganismen reiche Caecalflüssigkeit löst mehr Cellulose als die an Mikroorganismen arme; aber auch bei völliger Abwesenheit dieser werden noch gewisse Mengen von Cellulose gelöst. Die aus saurem Caecalinhalte gewonnene Flüssigkeit löst nur sehr wenig Cellulose. Einfaches Wasser und 1\% Sodalösung vermögen unter denselben Versuchsbedingungen Cellulose nicht

1) C. r. de la soc. de Biol. de Paris, 1901-1902. 
zu lösen, ebenso ist in den in Frage kommenden Nahrungsmitteln ein Cellulose lösendes Enzym nicht vorhanden. In den Extrakten und Sekreten der Caecalschleimhaut und der Caecaldrüsen ist ebenfalls ein Cellulose lösendes Enzym nicht zugegen.

Die Blinddarmflüssigkeit enthält ein proteolytisches, ein amylolytisches, ein Milchsäure- und ein invertierendes, aber kein lipolytisches Ferment.

Im Sekret bzw. Extrakt oder Preßsaft der Caecalschleimhaut ist dagegen kein proteolytisches Enzym vorhanden, wohl aber ein schwach wirkendes, saccharifizierendes Enzym. Dextrose wird in Milchsäure gespalten. Erepsin und Enterokinase sind darin nicht enthalten.

\section{Nachschrift.}

Die Untersuchungen von Bergmann (Skand. Arch. für Phys., Bd. XVIII, S. 119) sind mir leider erst nach der Absendung dieser Arbeit an die Redaktion bekannt geworden, weshalb dieselben im vorstehenden eine Berücksichtigung nicht erfahren haben. Scheunert. 